\title{
VARIA
}

\section{LA ESTANCIA ROMANA DEL PINTOR SEVILLANO DIEGO GUTIÉRREZ TERÁN AL SERVICIO DEL CARDENAL AGUSTÍN SPÍNOLA}

\author{
RAFAEL JAPÓN ${ }^{1}$ \\ Universidad de Granada \\ Università di Bologna
}

\begin{abstract}
El hallazgo de un documento relativo a la colección de pinturas reunida por el cardenal Agustín Spinola en Roma, fechado en 1633, permite situar en la Urbe en ese año al poco conocido pintor Diego Gutiérrez Terán, ignorándose hasta ahora su paso por Italia. Al mismo tiempo, dicho documento precisa cuáles fueron las iconografías de las pinturas reunidas por Spinola en Roma y traídas consigo a su vuelta a España.

Palabras clave: Diego Gutiérrez; Roma; Agustín Spinola; pintura italiana; Palacio Arzobispal de Sevilla; Academia de Murillo.
\end{abstract}

\section{THE ROMAN STAY OF THE SEVILLIAN PAINTER DIEGO GUTIÉRREZ TERÁN SERVING CARDINAL AGUSTÍN SPÍNOLA}

The discovery of a document relating to the collection of paintings owned by Cardinal Agustín Spinola in Rome, dated 1633, reveals that the little-known Sevillian painter Diego Gutiérrez Terán was in the city that year. His stay in Italy was ignored until now. The document likewise provides information concerning the subject matter and iconography of the paintings collected by Spinola in Rome and later taken with him on his return to Spain.

Key words: Diego Gutiérrez; Rome; Agustín Spinola; Italian painting; Archbishop's Palace of Seville; Murillo's Academy.

Cómo citar este artículo / Citation: Japón, Rafael (2019): "La estancia romana del pintor sevillano Diego Gutiérrez Terán al servicio del cardenal Agustín Spínola”. En: Archivo Español de Arte, vol. 92, núm. 366, Madrid, pp. 219-224. https://doi.org/10.3989/aearte.2019.15.

Desde que Diego Velázquez regresara a España tras su primer viaje a Italia, no se tenía constancia de la estancia en Roma de otro pintor sevillano hasta finales de la década de 1640 con Francisco de Herrera el Mozo. La influencia de la pintura italiana en la Sevilla del siglo XVII se desvela cada vez más clara entre los artistas que despuntaron hacia la mitad de la centuria, como se puede apreciar en la producción de Bartolomé Esteban Murillo, en la que abundan obras inspiradas en originales, copias y grabados italianos que llegaron a la capital hispalense por diferentes vías 2.

\footnotetext{
1 rafaeljaponf@gmail.com / ORCID iD: http://orcid.org/0000-0002-4203-2786.

2 Este artículo forma parte del proyecto de tesis La influencia de la pintura italiana en la escuela barroca sevilla$n a$, inscrita en la Universidad de Granada y en la Università di Bologna, dirigido por los profesores David García Cueto, Daniele Benati y Gabriele Finaldi.
} 
Como es conocido, este mismo maestro será uno de los promotores de la fundación de la Academia del Arte de la Pintura de Sevilla hacía 1658, y aunque parece que nunca realizó un viaje de formación fuera de los confines castellanos ${ }^{3}$, el arte proveniente de las más notables escuelas italianas estaría muy presente también en parte de su programa formativo ${ }^{4}$. La propia concepción de la Academia se ha comparado con aquellas otras fundaciones artísticas similares instauradas en Italia e imitada en el ámbito cortesano madrileño ${ }^{5}$, aunque la diferencia más notable es la financiación que, ajena a un patrocinio externo, era sustentada por los propios miembros ${ }^{6}$.

En las actas conservadas de las reuniones de esta institución se presentan una serie de nombres de mecenas y artistas — no solo pintores - ${ }^{7}$ que como miembros de la misma contribuían a la enseñanza y a la sustentación económica de la sede, situada en uno de los edificios más céntricos de la ciudad, la antigua Casa Lonja, hoy Archivo General de Indias. En los documentos de 1660 aparece presidiendo la Academia Francisco de Herrera el Mozo ${ }^{8}$, quien supuestamente demostró un elevado interés en ocupar este cargo antes de su viaje a la corte madrileña, haciendo con ello ostentación de la superioridad de su capacidad técnica gracias a su formación en Italia, vivencia que le diferenciaba de los demás miembros 9 .

Si bien están presentes pintores de gran relevancia, como los nombrados Murillo y Herrera, así como Juan de Valdés Leal o Pedro Núñez de Villavicencio, el análisis historiográfico realizado hasta la fecha sobre la mayoría de los pintores que firmaron las actas y otros documentos de la Academia es casi inexistente. Tal es el caso del pintor Diego Gutiérrez Terán, que más allá de su presencia en la institución entre 1669 y $1673^{10}$, solamente se conoce a través de ciertos instrumentos notariales de escaso interés histórico-artístico recopilados por Duncan T. Kinkead ${ }^{11}$. Pero es gracias a ellos que se tiene certeza del grado de madurez que alcanzaba como pintor hacia 1670, cuando en enero de ese mismo año firmaba un contrato como pintor de imaginería con el padre de un tal Francisco Pardo, comprometiéndose en la formación del joven ${ }^{12}$. En di-

\footnotetext{
3 Antonio Palomino relata que en los días que Murillo pasó en la corte, Velázquez lo persuadió para que realizara una estancia en Roma, aunque este viaje se truncó antes de su inicio al surgir problemas en la familia de Murillo que le obligaron a regresar a Sevilla (Palomino, 1986: 290-291). No obstante, su primer biógrafo Joachim von Sandrart afirmaba, pocos años después de la muerte del pintor, que sí llegó a formarse en Italia, donde incluso realizó varios encargos para diversos cardenales (Sandrart, 1683:397). Esta información no ha sido sostenida por la crítica posteriormente, debido a una supuesta fabulación de la vida del pintor por parte del autor alemán, quien introdujo un gran número de datos provenientes de su propia invención (Waterhouse, 1982: 101).

${ }^{4}$ Palomino, 1986: 312. Angulo Íñiguez, 1981: 101. La presencia del factor italiano llegó a ser física como recoge Palomino al hablar de la celosa competencia de Juan de Valdés Leal, miembro y presidente desde 1663 al 1666, al reconocer la superioridad técnica de un pintor italiano al que el Marqués de Villamanrique — protector de la institucióndio permiso para pintar en ella. Se desconoce de quién se trata, pero se sabe que una vez que terminó sus obras fueron expuestas en las cercanas gradas de la catedral obteniendo un rápido reconocimiento.

5 Aranda Bernal/Quiles García, 2000: 119-120. García Baeza, 2014: 119-126.

${ }^{6}$ Corzo Sánchez, 2009: 15. No obstante, el autor ve semejanzas con la Accademia degli Incamminati fundada por los Carracci en Bolonia, debido a que comparten la misma manera de sustentación económica y que en ambas el dibujo se ejercía a través de un modelo vivo. García Baeza, 2014: 118. En este sentido, la influencia de la pintura boloñesa en Sevilla ha sido estudiada por el que escribe estas líneas en Japón, 2018a: 27-38, 169-176. Sobre la academia de Murillo, véase también Cherry, 2002: 47-61.

7 Se conocen escultores y doradores, como Pedro Roldán o Bernardo Simón de Pineda. Véase, Banda y Vargas, 1982: 11-14. Corzo Sánchez, 2009: 67.

${ }^{8}$ Banda y Vargas, 1982: 24.

9 Ceán Bermúdez, 1806: 64-65. Corzo Sánchez, 2009: 15. En años posteriores, Pedro Núñez de Villavicencio, un pintor discípulo de Murillo que había conocido de primera mano el arte del sur de Italia en un viaje realizado a Malta, donde pudo formarse en el taller de Mattia Preti en 1662, así como en Roma en 1673, se erigió como presidente de la Academia. Véase González Ramos, 1999: 92-95.

10 Ceán Bermúdez, 1806: 153 y 160. Banda y Vargas, 1982: 23. Si bien Antonio García señala sólo su participación en 1673 (García Baeza, 2014: 246 y 269), se conoce que realizó pagos a la institución desde 1669 hasta 1674 (Kinkead, 2009: 238-239). Su figura se ignora en todos los estudios realizados sobre la pintura barroca española — véase Pérez Sánchez, 2010 - así como en aquellos especializados en el siglo XVII sevillano; véase Valdivieso, 2002; 2003.

11 Kinkead, 2009: 238-239.

12 Archivo Histórico Provincial de Sevilla (AHPS), Protocolos, oficio 17, 1670, I, 29. Kinkead, 2009: 238.
} 
versos poderes relativos a la gestión de propiedades y otros asuntos ajenos a su esfera profesional se reconoce como maestro del arte de pintor, hasta que se pierde su huella hacía 1693.

Aunque estas son las únicas noticias que se conocen de la vida de Diego Gutiérrez en Sevilla, se debe adscribir un importante pasaje a su biografía que puede revalorizar su figura dentro de la escuela sevillana. Un documento hallado en el Archivio Storico Capitolino de Roma sitúa al pintor residiendo en 1633 en aquella ciudad, desarrollando una actividad vinculada con su oficio en la morada de uno de los personajes de la facción española más interesantes en estas fechas entre ambas naciones, el cardenal Agustín Spinola (1597-1649). Por aquel entonces este eclesiástico de origen genovés, que era arzobispo de la diócesis de Santiago de Compostela y enviado especial de la monarquía hispánica en Roma donde ejercía el puesto de camarlengo del Sacro Colegio de Cardenales ${ }^{13}$, encomendó al artista sevillano la labor de inventariar los cuadros que había adquirido para la decoración del palacio en que residió en aquella ciudad desde 1630 al 1634. Se trataba de un procedimiento habitual en las ocasiones en que un extranjero debía trasladarse definitivamente de esta urbe y deseaba llevar consigo su colección artística ${ }^{14}$, como era el caso del propio Spinola, quien estaría en Madrid en septiembre del año sucesivo de 1634.

Si bien no se conoce desde cuándo se encontraba Diego Gutiérrez en Roma —ni si coincidió con Velázquez en su primer viaje realizado entre 1629 y $1631^{15}$-, por entonces ya firmaba como maestro pintor, y posiblemente estaría desarrollando una estancia formativa complementaría en Italia. Esta práctica instructiva en el seno del gremio de pintores sevillanos no debe de extrañar, puesto que era una experiencia deseada desde el siglo anterior entre los artistas de las principales escuelas españolas ${ }^{16}$, y especialmente en Sevilla las noticias sobre las novedades pictóricas debían de ser habituales, como consecuencia de la presencia de diversas familias de comerciantes llegadas de ciudades como Génova, Florencia o Roma, que deseaban tratar con América. En este sentido, Francisco Pacheco recogería para el Arte de la Pintura, publicado póstumamente en 1649, la transcendencia del viaje de aprendizaje mediante la información recibida de Pablo de Céspedes, que había trabajado en Roma en el círculo de Federico Zuccari y con Cesare Arbasia ${ }^{17}$, y especialmente de su yerno Diego Velázquez, quien lo habría informado de los detalles de su primera estancia romana $^{18}$. Tampoco consta la edad que alcanzaba Diego Gutiérrez en aquel momento ${ }^{19}$, y aunque es de suponer que debía ser un pintor joven, la tarea realizada para el cardenal Spínola requeriría de una cierta experiencia para reconocer y valorar las pinturas de otros maestros. Su estancia en Roma cobra mayor relevancia por medio de otros datos inéditos que señalan que dispuso su residencia en 1633 en un apartamento de la llamada Isola del Bufalo, nombre que hace referencia al palacio perteneciente a una familia de origen toscano de ese apellido. Aquella manzana formaba parte de la parroquia de Sant'Andrea delle Fratte, muy cercana a la embajada española, y en ella convivió con un personaje ilustre de la corte hispánica, el diplomático y escritor Diego Saavedra Fajardo (1584$1648)^{20}$, que estaba desempeñando desde 1631 la función de secretario de la misma embajada ${ }^{21}$.

13 Para mayor información sobre la estancia de Spinola en Roma, véase García Cueto, 2012: 1075-1076.

14 Otros ejemplos de este procedimiento notarial llevados a cabo en el siglo XVII se puede ver en Aterido Fernández, 2001: 179-181; así como Japón, 2018b: 82-95.

15 Sobre esta primera estancia velazqueña en Italia, véase Salort Pons, 2002: 31-56.

16 Checa Cremades, 2007: I-IV.

17 Baglione, 1733: 19. Pacheco, 1985: 101. Díaz Cayeros, 2000: 11-15.

18 Pacheco, 2001: 206-209.

19 Los documentos recopilados por Duncan T. Kinkead no proporcionan ninguna información al respecto, más allá de un extracto donde parece especificar las edades de él y su mujer, siendo totalmente incoherentes, ya que este dato se debe a otro poder anterior citado en 1670 del que no se detalla la fecha concreta de redacción. Además, en él no se puntualiza la profesión de Diego Gutierrez, pudiéndose confundir con un personaje homónimo, por lo que se ha decidido no tener en cuenta este apunte biográfico hasta una confirmación más exacta. Véase Kinkead, 2009: 238; AHPS, Protocolos, oficio 17, 1670, I, 13-14.

${ }^{20}$ Archivio del Vicariato di Roma (ASVR), Sant'Andrea delle Fratte, State d'Anima, 6, 1633, f. 246v. Se reconoce como "Diego pittore spagnolo". Por esta misma fuente se sabe también que en una vía cercana a su residencia, en la Isola della Strada della Vita, vivió en aquel mismo año otro personaje sevillano, un sacerdote llamado Alonso de Rojas.

${ }^{21}$ Clarkson Dowling, 1977: 19. 
En el inventario se enumeran más de treinta obras de las que solo se refiere su iconografía, siempre religiosa, siendo en su mayoría efigies de santos, escenas de la vida de Cristo y de la Virgen, y dos pinturas relativas a personajes del Antiguo Testamento, Job y el rey David ${ }^{22}$. Si bien para ninguna de ellas se refleja el nombre de su autor, serían probablemente todas adquiridas en el mercado romano en los años que el cardenal estuvo residiendo allí con el objetivo de servir a la decoración de su apartamento, como se refleja en el poder citado. No obstante, gracias al testamento del cardenal y de su sobrino y protegido, Ambrosio Ignacio Spinola — quien también llegaría a ser arzobispo de Sevilla-, se puede confirmar que estas obras fueron enviadas a España y acompañaron a su dueño en su última morada, el Palacio Arzobispal hispalense. Algunas de ellas son mencionadas en su testamento, fechado en Sevilla el 22 de septiembre de 1647. De esta manera se deduce que en la colección romana se exhibían cuadros de grandes artistas, como el caso del primero que se inventaría, La oración en el huerto, que se puede identificar con una pintura realizada por los Bassano, habiendo pertenecido posteriormente a su sobrino Ambrosio de Spínola, quien a su vez lo dejaría en herencia a su prima la condesa de $A r \cos ^{23}$. Se hallaba también una obra de Tiziano representando al Salvador, que en el inventario fechado en 1633 viene descrito como una testa del Salvador, la cual pasó a la marquesa de Leganés ${ }^{24}$. Del mismo pintor parece que existió el original, o quizás una copia de la Virgen del conejo ejecutada en origen para el Duque de Mantua, que el propio Agustín dejó en su testamento a Filippo Spinola ${ }^{25}$, y con mucha probabilidad corresponde con Un desposorio de santa Catherina que se puede contemplar en el inventario analizado en esta ocasión. Por último, se conoce que la Concepción de Nuestra Señora fue realizada en Roma en un gran formato por Tommaso Dovini, el Caravaggino, gracias a un pleito en que estuvo involucrado Fernando Álvarez, uno de los mayordomos del cardenal que se encargó de recoger el cuadro en el taller del maestro italiano, en cuyo estilo se advierte varias fuentes, siendo las más evidentes el caravaggismo, así como el clasicismo boloñés a través de Giovanni Lanfranco ${ }^{26}$.

Para 1679, cuando se fecha el testamento de su sobrino Ambrosio, los demás cuadros de origen italiano habrían acabado dispersos en otras colecciones, según dispuso su tío antes de fallecer, haciendo dificultosa la identificación del elenco completo. Sin embargo, cabe pensar que con la llegada de Agustín Spinola a Sevilla en 1645, todos los lienzos descritos en la lista se expusieran en el Palacio Arzobispal, pues el mismo cardenal decidió decorar las galerías de la residencia con su propia colección, acrecentándola además con obras de artistas locales, como Murillo — quien llevaría a cabo un retrato del marqués de Leganés, su sobrino ${ }^{27}$ - Pablo Legot - quien realizaría un Apostolado de cuerpo entero-, Juan de Zamora — que pintó dieciseises lienzos con escenas bíblicas - y Francisco Herrera, que hizo otros cuatro cuadros de grandes dimensiones ${ }^{28}$. Por tanto, es posible suponer que algunas de las pinturas del Palacio Arzobispal de Sevilla que aún siguen sin autor fueran de Diego Gutierrez, puesto que el nombramiento del cardenal Spínola como arzobispo de la diócesis hispalense le llevaría a vivir en la ciudad hasta su muerte en 1649, tiempo en el que seguramente el pintor le ofrecería sus servicios, reencontrándose con él tras la estancia de ambos en Roma. Además, en aquellos años decidió reformar

22 Inventario de las pinturas reunidas en la residencia del cardenal Agustín Spinola en Roma, 11 de abril de 1633, Archivio Storico Capitolino di Roma (ASCR), Archivio Urbano, Sezione I, 520, s.p. Véase apéndice documental.

${ }^{23}$ Quiles García, 2011: 740. El autor duda si éste lienzo pudo pertenecer a Agustín Spínola antes de entrar en la colección de su sobrino, información que se confirmaría con este dato.

24 Quiles García, 2011: 741. A su sobrino el marqués de Leganés mandó un retrato de su padre, también marqués de la misma estirpe, realizado por Van Dick. Este cuadro estuvo en el Palacio Arzobispal de Sevilla, pero no proviene de la colección pictórica creada por el cardenal en Roma.

25 Quiles García, 2011: 740-741. Como bien refiere el autor, este mismo cuadro volvió a Sevilla de manos de Ambrosio Spinola, que en 1679 lo dejó en herencia a su hermana la marquesa de Almazán.

${ }^{26}$ Curti, 2003: 153-156. En el proceso judicial, el Caravaggino responde a la pregunta del juez que requiere mayores datos sobre el encargo de Spinola, contestando que "il quadro che gli feci era una Concetione della Madonna in grande".

27 Quiles García, 2011: 742.

28 Valdivieso/Serrera, 1979:8, 58-59 y 65-70. Las únicas obras conservadas in situ son aquellas salidas del taller de Juan de Zamora. 
el palacio ${ }^{29}$, en cuyo jardín mandó realizar una gruta al estilo de la existente en los Reales Alcázares y la colocación de una fuente de mármol, rematada con un grupo escultórico que representa a Hércules y el león de Nemea, creada en talleres genoveses ${ }^{30}$.

Gutiérrez tendría, por tanto, una carrera larga y fructífera, pues las últimas noticias que se tiene de él lo sitúan en Sevilla en las postrimerías del siglo. Sin embargo, paradojas de la historia, no se conoce ninguna obra de su producción actualmente debido al desconocimiento de los rasgos de su estilo. Aún así, se muestra como un artista cuya formación en Sevilla y en Roma se da paralelamente a la de Diego Velázquez, y su presencia en la Academia de pintura, como en su propio taller, supondría un impulso a la difusión de los modelos italianos que, como se ha demostrado, pudo estudiar de la mano de notables mecenas del siglo XVII como el cardenal Agustín Spinola y el secretario de la embajada de Felipe IV Diego Saavedra Fajardo.

\section{APÉNDICE DOCUMENTAL}

Doc.1. Inventario de los cuadros de la residencia romana del cardenal Agustín de Spínola. 11 de abril de 1633. Archivio Storico Capitolino, Archivio Urbano, Sezione I, 520, s.p.

In Dei nomine Amen. Sepan quantos, como yo Jayme Morer por autoridad apostólica notario público infraescrito insstado y requerido por parte y a instancia del señor don Agustín Spinola canónigo y dignidad que llaman cardenal de las iglesias metropolitanas compostellana, me fui al palacio del eminentísimo y reverendísimo don Agustín Spíniola arçobispo compostellano, cuyo gentilhombre es el dicho señor don Agustín y ocularmente vi que por su devoción y por ornato del aposento en que dicho señor don Agustín vive y mora en dicho palacio tenía y tiene colgados y puestos los quadros y efigies siguientes:

La oración del huerto, la Concepción de Nuestra Señora, san Jerónimo, san Sebastián, san Sebastián, Jusepe con el Niño Jesús y la cabeza de San Juan, san Juan, san Miguel y san Francisco de imagen entera al natural. Un desposorio de santa Catherina, nuestra señora del Rosario, san Antonio y san Pablo primer eremita, nuestra señora con el Jesús y san Joseph, una nuestra señora circuida de rosas, una cabeça de san Juan, nuestra señora de la Soledad, una testa de nuestra señora con el niño Jesús, un ecce homo, un san Francisco, otro san Josepe pequeño con el niño durmiendo, una testa del Salvador, un Job con su mujer, un quadro de un ermitaño que es una tentación de noche, el prendimiento de Christo, una testa de san Pedro, un san Antonio pequeño en rame con tres aros o cornisas doradas de diferentes grandezas, y quatro Christos vivos pequeños, es a saber tres en lámina con sus guarniciones, y otro en leño, y un quadrito de David muy pequeño.

Y para que conste desta verdad, y que dichos quadros han servido y sirven para el dicho o dichos effetos hize de ello a dicha instancia el presente instrumento público, que fue hecho en Roma en el palacio de dicho eminentísimo cardenal que es en la calle del corso, siendo presentes por testigos llamados y rogados que juntamente conmigo vieron los quadros, el señor don Thomás de Monrroy clérigo del obispado de Zamora, y el señor licenciado Gaspar Piquero de Meneses, obispado de Oviedo, su eminencia y Diego Gutiérrez pintor de la ciudad de Sevilla residentes en Roma.

\section{BIBLIOGRAFÍA}

Angulo Íñiguez, Diego (1981): Murillo, tomo I. Madrid: Espasa-Calpe.

Aranda Bernal, Ana María/Quiles García, Fernando (2000): "Las Academias de pintura en Sevilla”. En: Academia: Boletín de la Real Academia de Bellas Artes de San Fernando, 90, Madrid, pp. 119-138.

Aterido Fernández, Ángel (2001): “De Castellón a Roma: el canónigo Vicente Giner (ca. 1636-1681)”. En: Archivo Español del Arte, 294, Madrid, pp. 179-183.

Baglione, Giovanni (1723): Le vite de' pittori, scultori et architetti dal ponteficato di Gregorio XIII del 1572 in fino a tempi di Papa Urbano Ottavo nel 1642. Nápoles: [s.e].

Banda y Vargas, Antonio de la (1982): El manuscrito de la Academia de Murillo. Sevilla: Confederación Española de Centros de Estudio.

\footnotetext{
29 Falcón Marques, 1997: 56-61.

${ }^{30}$ Quiles García, 2011: 738. García Cueto, 2012: 1078.
} 
Ceán Bermúdez, Juan Agustín (1806): Carta a un amigo suyo sobre el estilo y gusto en la pintura de la escuela sevillana; y sobre el grado de perfección a que la elevó Bartolomé Esteban Murillo: cuya vida se inserta, y se describen sus obras en Sevilla. Cádiz: Casa de la Misericordia.

Checa Cremades, Fernando (2007):“Aprendizaje y triunfo: el viaje del artista y la difusión de nuevos modos estéticos en la Edad Moderna”. En: Checa Cremades, Fernando (dir.): El viaje del artista en la Edad Moderna: materiales para su estudio. Madrid: Editorial Complutense, pp. I-IV.

Cherry, Peter (2002): “Murillo's drawing Academy”. En: Stratton-Pruitt, Suzanne L. (coord.): Bartolomé Esteban MuriIlo (1617-1682): paintings from american collections. Nueva York: Harry N. Abrams, pp. 47-61.

Clarkson Dowling, John (1977): Diego de Saavedra Fajardo. Boston: Twayne Publishers.

Corzo Sánchez, Ramón (2009): La Academia del Arte de la Pintura de Sevilla: 1660-1674. Sevilla: Instituto de Academias de Andalucía.

Curti, Francesca (2003): "Precisazioni documentarie su Tommaso Dovini detto il Caravaggino". En: Cappelletti, Francesca (coord.): Decorazione e collezionismo a Roma nel Seicento. Roma: Gangemi, pp. 141-157.

Díaz Cayeros, Patricia (2000): "Pablo de Céspedes entre Italia y España". En: Anales del Instituto de Investigaciones Estéticas, 76, México, pp. 5-60.

Falcón Márquez, Teodoro (1997): El Palacio Arzobispal de Sevilla. Córdoba: CajaSur, Obra Social y Cultural.

García Baeza, Antonio (2014): Entre el obrador y la academia. La enseñanza de las artes en Sevilla durante la segunda mitad del Seiscientos. Sevilla: Instituto de la Cultura y las Artes de Sevilla.

García Cueto, David (2012): "El mecenazgo episcopal de Agustín Spínola (1597-1649)”. En: Barral Rivadullo, María Dolores et al. (coord.): Mirando a Clío: el arte español espejo de su historia: actas del XVIII Congreso del CEHA. Santiago de Compostela: Universidad de Santiago de Compostela, pp. 1071-1085.

González Ramos, Roberto (1999): Pedro Núñez de Villavicencio: Caballero Pintor. Sevilla: Diputación Provincial de Sevilla.

Japón, Rafael (2018a): "La presenza della pittura bolognese a Siviglia: collezionismo e influssi". En: Pigozzi, Marinella (coord.): Dialogo artistico tra Italia e Spagna. Arte e música. Bolonia: Bononia University Press, pp. 27-38, 169-176.

Japón, Rafael (2018b): “Copias sacras y originales profanos: la colección pictórica del arcediano Domingo de Mendiola en el primer tercio del Seiscientos en Roma". En: Revista de História da Arte. Série W, Lisboa, pp. 82-95.

Kinkead, Duncan T (2009): Pintores y doradores en Sevilla 1650-1699: documentos. Bloomington: Authorhouse.

Mena Marqués, Manuela B. (coord.) (1983): Bartolomé Esteban Murillo (1617-1682) [Exposición]. Madrid: Fundación Juan March.

Pacheco, Francisco (Bassegoda, Bonaventura ed.) (2001): Arte de la pintura. Madrid: Cátedra.

Pacheco, Francisco (Piñero, Pedro M. y Reyes, Rogelio eds.) (1985): Libro de descripción de verdaderos retratos de ilustres y memorables varones. Sevilla: Diputación Provincial.

Palomino, Antonio (Ayala Mallory, Nina ed.) (1986): Vidas. Madrid: Alianza Editorial.

Pérez Sánchez, Alfonso E. (Navarrete Prieto, Benito (ed.) (2010): Pintura barroca en España (1600-1750). Madrid: Cátedra.

Quiles García, Fernando (2011): "El arzobispo Agustín Spínola, promotor de las artes sevillanas del barroco (1645-1649)". En: Herrero Sánchez, Manuel et al. (coord.): Génova y la monarquía hispánica (1528-1713). Genova: Società Ligure di Storia Patria, pp. 731-752.

Salort Pons, Salvador (2002): Velázquez en Italia. Madrid: Fundación de Apoyo a la Historia del Arte Hispánico.

Sandrart, Joaquim von (1683): Academia nobilissimae artis pictoriae. Nuremberg: Froberger.

Valdivieso, Enrique (2002): Historia de la pintura sevillana: siglos XIII al XX. Sevilla: Guadalquivir.

Valdivieso, Enrique (2003): Pintura barroca sevillana. Sevilla: Guadalquivir.

Valdivieso, Enrique/ Serrera, Juan Miguel (1979): Catálogo de las pinturas del Palacio Arzobispal de Sevilla. Sevilla: los autores.

Fecha de recepción: 18-II-2018

Fecha de aceptación: 29-I-2019 